\title{
GMR
}

\section{Correlation between non-metastatic protein 23 expression and clinicopathological features of colorectal cancer in Asians}

\author{
J.W. Fu and X.Q. Chu \\ Department of Gastrointestinal Surgery, Shandong Jining No.1 People's Hospital, \\ Shandong, China \\ Corresponding author: X.Q. Chu \\ E-mail: chuxianqun711@126.com \\ Genet. Mol. Res. 14 (4): 15597-15608 (2015) \\ Received August 6, 2015 \\ Accepted October 22, 2015 \\ Published December 1, 2015 \\ DOI http://dx.doi.org/10.4238/2015.December.1.11
}

ABSTRACT. The current meta-analysis was performed to investigate the association between non-metastatic protein 23 (NM23) expression, tumor pathology, and disease prognosis in colorectal cancer (CRC) among Asians. English and Chinese language-based electronic databases (e.g., PubMed, EBSCO, Ovid, Springerlink, Wiley, Web of Science, Wanfang databases, China National Knowledge Infrastructure, VIP databases) were searched using search terms to identify published studies relevant to NM23 and CRC with immunohistochemistry. In total, 289 studies were identified through database searches, and 16 cohort studies (4 studies in English, 12 in Chinese) were chosen for meta-analysis, which included 1592 CRC patients. The results revealed that NM23 protein expression in CRC tissue was higher in patients with Dukes stages A and B than in patients with Dukes stages $C$ and D. The NM23 protein was expressed at higher levels in well- and moderately differentiated tumors than in poorly differentiated tumors. The 5-year survival rate was also higher in CRC patients with NM23-positive tumors than in CRC patients with NM23-negative tumors. Significantly, 5-year tumor relapse and metastasis were lower in patients with NM23-positive tumors than in CRC patients with NM23-negative 
tumors. The findings suggest that NM23 expression status is associated with tumor aggressiveness and survival in CRC among Asians. Importantly, CRC patients with NM23-positive tumors had a better prognosis, and thus NM23 expression maybe used as a key prognostic indicator for CRC.

Key words: Colorectal cancer; Meta-analysis; Non-metastatic protein 23; Pathological characteristics; Prognosis

\section{INTRODUCTION}

Colorectal cancer (CRC) accounts for approximately 1.25 million newly diagnosed cases each year and is responsible for more than 600,000 cancer-related mortality worldwide (Qu et al., 2013). It is the third leading cause of cancer deaths in the US and the third most common cancer in the US and UK, with over 37,000 new diagnoses in the UK each year (Siegel et al., 2014). The highest rates are observed in developed countries such as Australia, New Zealand, Europe, and the US, while low rates are found in Africa and south-central Asia (Merika et al., 2010). The symptoms and signs of CRC vary depending on the location of the tumor and if metastasis has occurred. General symptoms include rectal bleeding, diarrhea, constipation, loss of weight, abdominal pain, and anemia (Astin et al., 2011; John et al., 2011). CRC staging is conducted based on the TNM system or Dukes staging, which convey the local extent of tumor, lymphatic spread, venous spread, and histologic grading. Typically, for Dukes stage A, the cancer is only present in the inner lining of the bowel; in Dukes stage B, the cancer has invaded the muscle; in Dukes stage $C$, the cancer has invaded the nearby lymph nodes; in Dukes stage D, the cancer has metastasized (Obrocea et al., 2011; linuma et al., 2011). Approximately 75-95\% of CRC cases are associated with lifestyle risk factors, aging, and underlying genetic risks (Cunningham et al., 2010; Watson and Collins, 2011). It is thought that the Ang-2, Tie-2, PI3K, and AKT genes are correlated with differentiation level, stage of Dukes classification, and lymphatic metastasis in CRC (Zhang et al., 2014). CRCs are aggressive, and $20-50 \%$ of CRC patients die within 5 years after diagnosis because of extensive metastatic disease spread (Tan et al., 2012). Furthermore, relapse and metastasis are the leading causes of deaths in CRC, with greater than $50 \%$ of CRC patients developing metastases with unresectable tumors (Qu et al., 2013). Therefore, significant efforts have been directed towards early detection and biomarker discovery for CRC (Jimenez et al., 2010).

Non-metastatic gene 23 (NM23) (encoded by NME1), was discovered because of its reduced expression in a comparative hybridization screen of poorly versus highly metastatic sublines of the murine melanoma cell line K-1735 in 1988 and is the first metastasis suppressor gene discovered fromover 20 metastasis suppressor genes known to date (Marshall et al., 2010). NM23 is thought to have a dual role, as its expression is induced in primary cancers, but its loss of expression at later stages promotes tumor progression (Saha and Robertson, 2011). Multiple biochemical functions have been identified for NM23 proteins, and several may have anti-metastatic properties (Steeg et al., 2011). Cell lines from multiple histological types of cancer were suppressed from undergoing metastasis by NM23. However, no studies have shown that overexpression of NM23 completely abrogated metastasis (Marino et al., 2012). Tumor metastasis is a complex multistep process and involves alterations in multiple genes that influence the spread of tumor cell origin sites to distant metastatic sites in the body. The process is characterized by series of events such as activation/inactivation of metastasis-regulating genes, altered cell adhesion, increased cell 
motility, expression of protein hydrolases, and formation of new blood vessels (Tan et al., 2012; Qu et al., 2013). NM23-H1 was the first identified metastasis suppressor gene and belongs to a group of 8 closely related genes, $\mathrm{Nm} 23 \mathrm{H} 1-\mathrm{H} 8$. These $\mathrm{NM} 23$ genes are likely regulated independently because NM23-H4, NM23-H6, and NM23-H7 were found to be overexpressed in gastric cancer and CRC (Lu et al., 2013). The exact mechanism for the origin and development of CRC remains unclear. Previous studies have shown that inactivation of NM23 plays an important role in the development of CRC (Wu et al., 2013; Qu et al., 2013). We explored the correlation between NM23 expression, tumor pathology, and tumor prognosis to better understand the influence of NM23 status on CRC progression and survival.

\section{MATERIAL AND METHODS}

\section{Literature search}

To identify all relevant studies that assessed the correlation between NM23 expression and CRC, we comprehensively searched the PubMed, Web of Science, Cochrane Library, CISCOM, CINAHL, China Bio Medicine, VIP, Wanfang, and China National Knowledge Infrastructure databases (last updated search in September 2014) using search terms related to CRC and NM23. The search terms included the following: ("Colorectal Neoplasms" or "Colonic Neoplasms" or "Rectal Neoplasms" or "Cecal Neoplasms" or "Colorectal Tumor" or "Colonic Tumor" or "Rectal Tumor" or "Cecal Tumor" or "Colorectal Cancer" or "Colonic Cancer" or "Rectal Cancer" or "Cecal Cancer") and ("NM23" or "NM23 Protein"). We further scanned the bibliographies of relevant articles manually to identify additional relevant papers.

\section{Study selection}

Selected studies were included in the meta-analysis if they met the following inclusion criteria: 1) clear data were presented and related to an association between NM23 expression, pathological characteristics, and prognosis of $\mathrm{CRC}$; 2) the patients had a confirmed diagnosis of $\mathrm{CRC} ; 3)$ the studies contained the required statistical data; 4) the language was restricted to Chinese and English. The major exclusion criteria were as follows: 1) insufficient information; 2) duplicate publications; 3) unclear diagnostic criteria for CRC patients.

\section{Data extraction and quality assessment}

Data were extracted from the selected studies by 2 independent investigators. Information collected included the following: surname and initials of the first author, year of submission, country, study design, gender, demographic variables, detection method of NM23 expression, and NM23 expression levels, among other points. The 2 investigators assessed the quality of the cohort trials using the Newcastle-Ottawa Scale (NOS) criteria (Stang, 2010). The NOS criteria are as follows: 1) representativeness of the exposed cohort; selection of the non-exposed cohort; ascertainment of exposure; demonstration that the outcome of interest was not present at the start of study; 2) whether the study was selected and analyzed according to the most important factor; whether the study controlled other confounding factors; 3) whether follow-up was long enough for outcomes to occur; adequacy of follow-up of cohorts. Disagreements between the 2 independent investigators were resolved by a third investigator. 


\section{Statistical analysis}

The meta-analysis was conducted using STATA 12.0 (Stata Corp., College Station, TX, USA). The association between NM23 expression, tumor pathology, and prognosis of CRC was estimated by the standardized mean difference and $95 \% \mathrm{Cl}$. We used Cochran's Q-statistic $\left(P_{h}<0.05\right.$ was considered to be significant) and $l^{2}$ tests to quantify heterogeneity among studies (Zintzaras and Ioannidis, 2005b). In order to calculate the pool standardized mean differences, the fixed-/randomeffect models were used; the random-effect model was applied when significant heterogeneity was observed $\left(P_{h}<0.05\right.$ or $l^{2}$ test exhibited $\left.>50 \%\right)$, whereas standardized mean differences were pooled based on the fixed-effect model (Higgins and Thompson, 2002; Zintzaras and loannidis, 2005a). In addition, the effect of publication bias was detected by the Egger linear regression test $(P<0.05$ was considered to be significant), which can be used to evaluate the funnel plot asymmetry, revealing possible publication bias (Song and Gilbody, 1998; Peters et al., 2006).

\section{RESULTS}

\section{Demographic variables}

Figure 1 shows the flow chart of study selection based on exclusion criteria. First, 289 potential articles were identified from the databases using search terms and manual review. Of the 289 articles, 94 studies were duplicates and were removed. After screening of the title and abstract, 154 studies were found to be irrelevant and subsequently excluded. Of the remaining studies, 20 were excluded after more detailed reading of the full text. Qualitative analysis was performed on 21 studies selected. Finally, 16 studies published from 1999-2014 were incorporated into our meta-analysis (Feng et al., 1999; He et al., 2000; Yu et al., 2000; Liu and Wang, 2001; Wang et al., 2001; Zhen et al., 2002; Zhang et al., 2003; Su and Li, 2004; Liu et al., 2005; Ba, 2006; Gao, 2006; Chen et al., 2007; Song et al., 2010; Wu et al., 2013; Qu et al., 2013; Cui et al., 2014). The baseline characteristics and NOS scores of the 16 eligible studies are summarized in Table 1 and Figure 2, respectively.

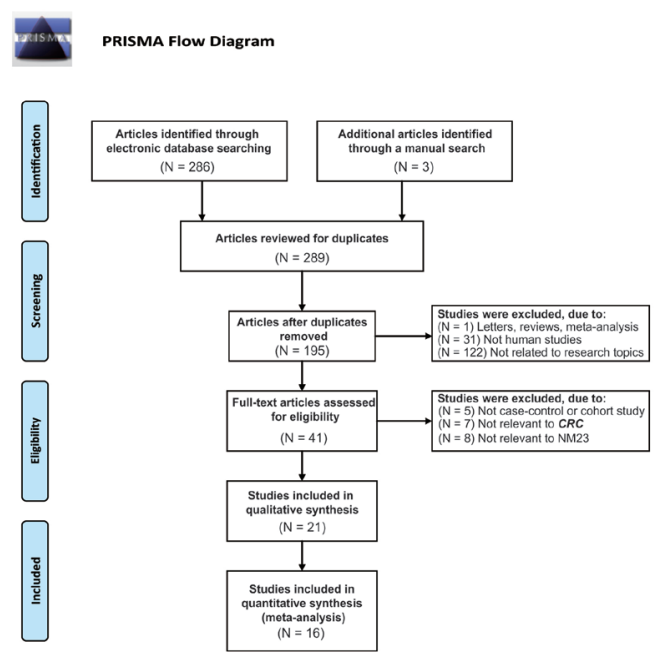

Figure 1. Flow chart shows the study selection procedure. Sixteen studies were included in the meta-analysis. 


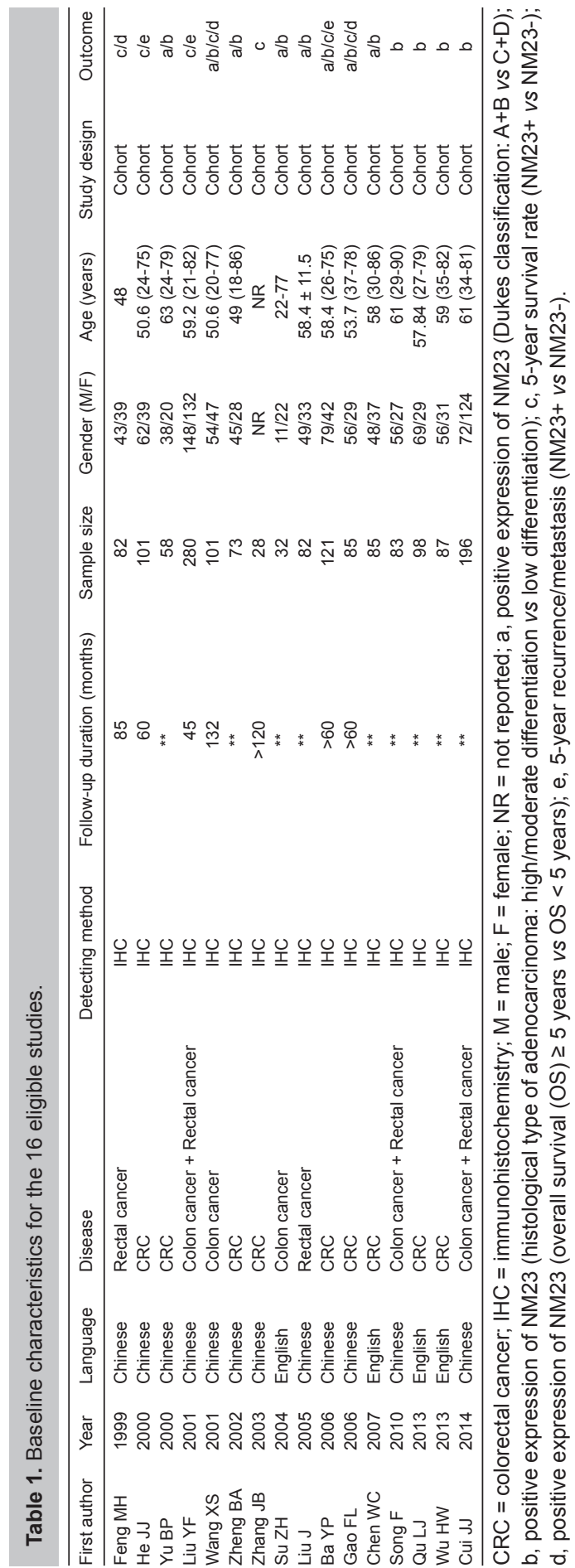




\begin{tabular}{|c|c|c|c|c|c|c|c|c|c|c|}
\hline & & $\begin{array}{l}5 \\
\text { ஸ } \\
\text { ○ }\end{array}$ & $\begin{array}{l}\cong \\
\mathscr{D} \\
\text { O }\end{array}$ & \begin{tabular}{l}
$m$ \\
0 \\
0 \\
\hdashline
\end{tabular} & $\begin{array}{l}\text { オ } \\
\text { હ } \\
\text { O }\end{array}$ & $\begin{array}{l}\text { D } \\
\text { ஹ } \\
0 \\
Z\end{array}$ & $\begin{array}{l}\mathscr{0} \\
\text { ஹ } \\
0 \\
Z\end{array}$ & $\begin{array}{l}\hat{O} \\
\text { ஸ } \\
0 \\
Z\end{array}$ & $\begin{array}{l}\infty \\
0 \\
\mathcal{O} \\
\text { O }\end{array}$ & $\begin{array}{l}\text { O } \\
\text { ஹ } \\
\text { ○ }\end{array}$ \\
\hline Feng MH & (1999) & + & + & ( & + & + & + & $?$ & 4 & + \\
\hline He JJ & (2000) & . & ) & & & + & 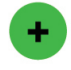 & & & + \\
\hline Yu BP & (2000) & + & & & & + & ) & $?$ & & + \\
\hline Liu YF & (2001) & + & + & + & + & + & + & $?$ & & + \\
\hline Wang XS & $(2001)$ & + & 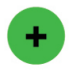 & & & + & + & & & \\
\hline Zheng BA & (2002) & + & & & & +1 & + & $?$ & & \\
\hline Zhang JB & (2003) & 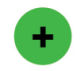 & & & & - & ) & $?$ & & \\
\hline Su ZH & (2004) & + & + & (. & + & + & + & & & \\
\hline Liu J & (2005) & + & & & & $t$ & . & & & \\
\hline $\mathrm{Ba}$ YP & (2006) & + & + & . & + & + & + & $?$ & & \\
\hline Gao FL & (2006) & + & + & . & + & ) & ( & $?$ & & \\
\hline Chen WC & (2007) & + & + & ( & + & + & + & & & \\
\hline Song $\mathrm{F}$ & (2010) & + & + & & & + & 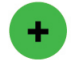 & ? & & \\
\hline Qu LJ & (2013) & + & + & $(t$ & + & 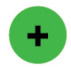 & (t) & & & \\
\hline Wu HW & (2013) & + & $\Delta$ & . & + & 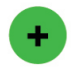 & + & & $?$ & \\
\hline Cui JJ & (2014) & + & + & & . & -) & + & & $?$ & \\
\hline
\end{tabular}

Figure 2. Newcastle-Ottawa Scale scores for the 16 eligible studies. 


\section{Meta-analysis of association between NM23 protein expression and CRC pathological characteristics}

The correlation between NM23 protein expression and the pathological features of CRC was investigated in 12 studies. Heterogeneity was observed and thus the random-effect model was applied in the present study (Dukes staging: $I^{2}=53.613 \%, \mathrm{P}_{\mathrm{h}}=0.035$; histological types: $I^{2}=$ $\left.59.846 \%, P_{h}=0.004\right)$. Higher NM23 protein expression was observed in patients with Dukes stage $A$ and $B$ than in patients with Dukes stage $C$ and $D(R R=1.303,95 \% C l=1.129-1.504, P<0.001)$. In addition, the NM23 protein was expressed at a higher level in well- and moderately differentiated tumors than in poorly differentiated tumors $(R R=1.369,95 \% \mathrm{Cl}=1.145-1.637, \mathrm{P}=0.001)$ (Figure 3).

\section{A}

Positive Expression (Dukes' classification) (NM23+ vs NM23-)

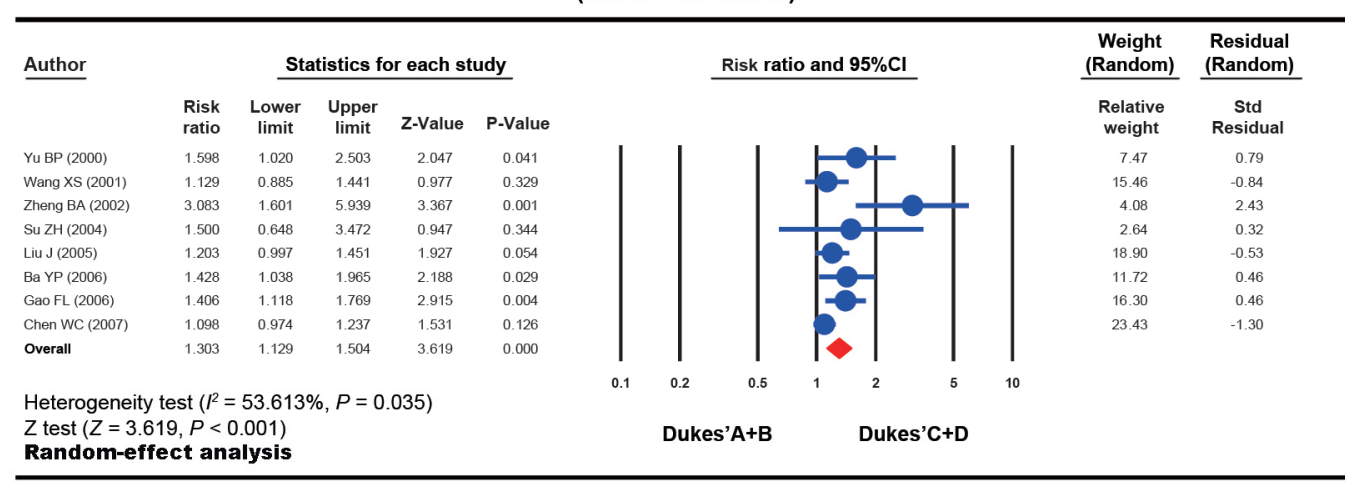

B

Positive Expression (Histological Type of adenocarcinoma) (NM23+ vs NM23-)

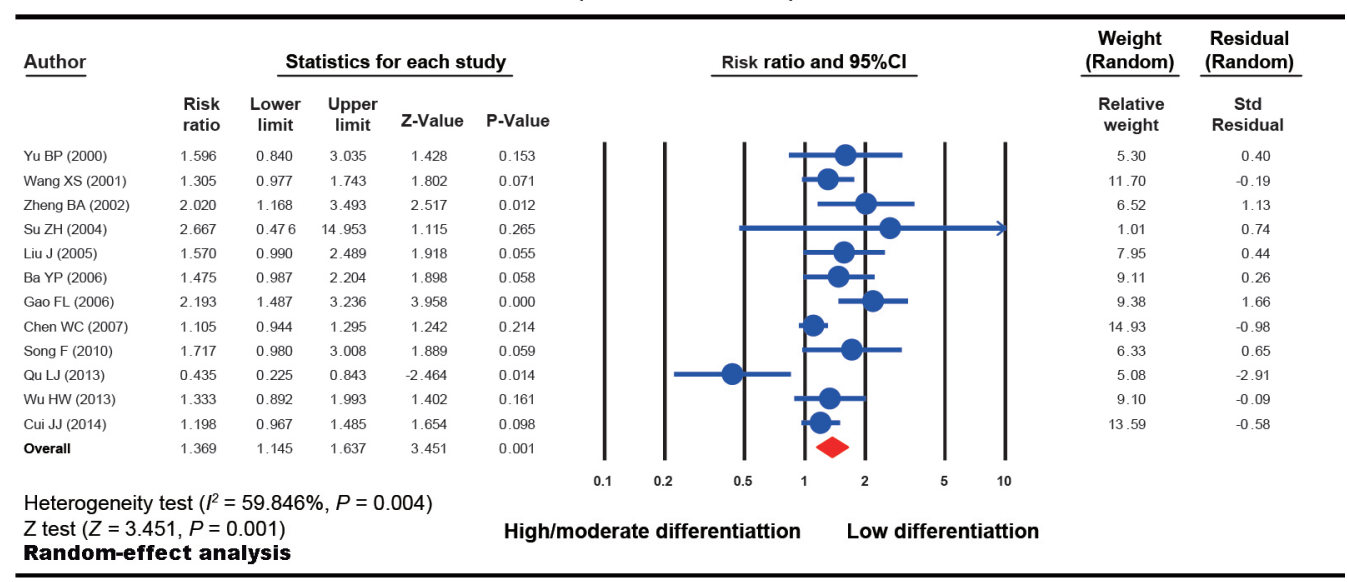

Figure 3. Forest analyses for the correlation between NM23 expression and pathological characteristics of colorectal cancer among Asians. 


\section{Meta-analysis of association between NM23 protein expression and CRC prognosis}

The correlation between NM23 protein expression and CRC prognosis was reported in 7 studies. No heterogeneity was found in the 5-year survival rate and 5-year tumor relapse and the occurrence of metastasis (5-year survival rate: $I^{2}=19.737 \%, \mathrm{P}_{\mathrm{h}}=0.279 ; 5$-year tumor relapse and metastasis: $l^{2}=3.76 \%, \mathrm{P}_{\mathrm{h}}=0.354$ ); therefore, the fixed-effect model was applied. However, the random-effect model was used when heterogeneity was observed for the positive expression of the NM23 protein before and after 5 -year survival $\left(I^{2}=93.585 \%, \mathrm{P}_{\mathrm{h}}<0.001\right)$. The results showed that the 5-year survival rate was higher in NM23-positive CRC patients than in NM23-negative CRC patients $(\mathrm{RR}=1.862,95 \% \mathrm{Cl}=1.560-2.222, \mathrm{P}<0.001)$. Five-year tumor relapse and metastasis rates were lower in NM23-positive CRC patients than in NM23-negative CRC patients $(R R=0.516$, $95 \% \mathrm{Cl}=0.419-0.634, \mathrm{P}<0.001)$. However, there was no significant difference in NM23 protein expression between CRC patients with $\geq 5$-year survival and CRC patients with $<5$-year survival $(\mathrm{RR}=1.139,95 \% \mathrm{Cl}=0.960-1.352, \mathrm{P}=0.271)($ Figure 4$)$.

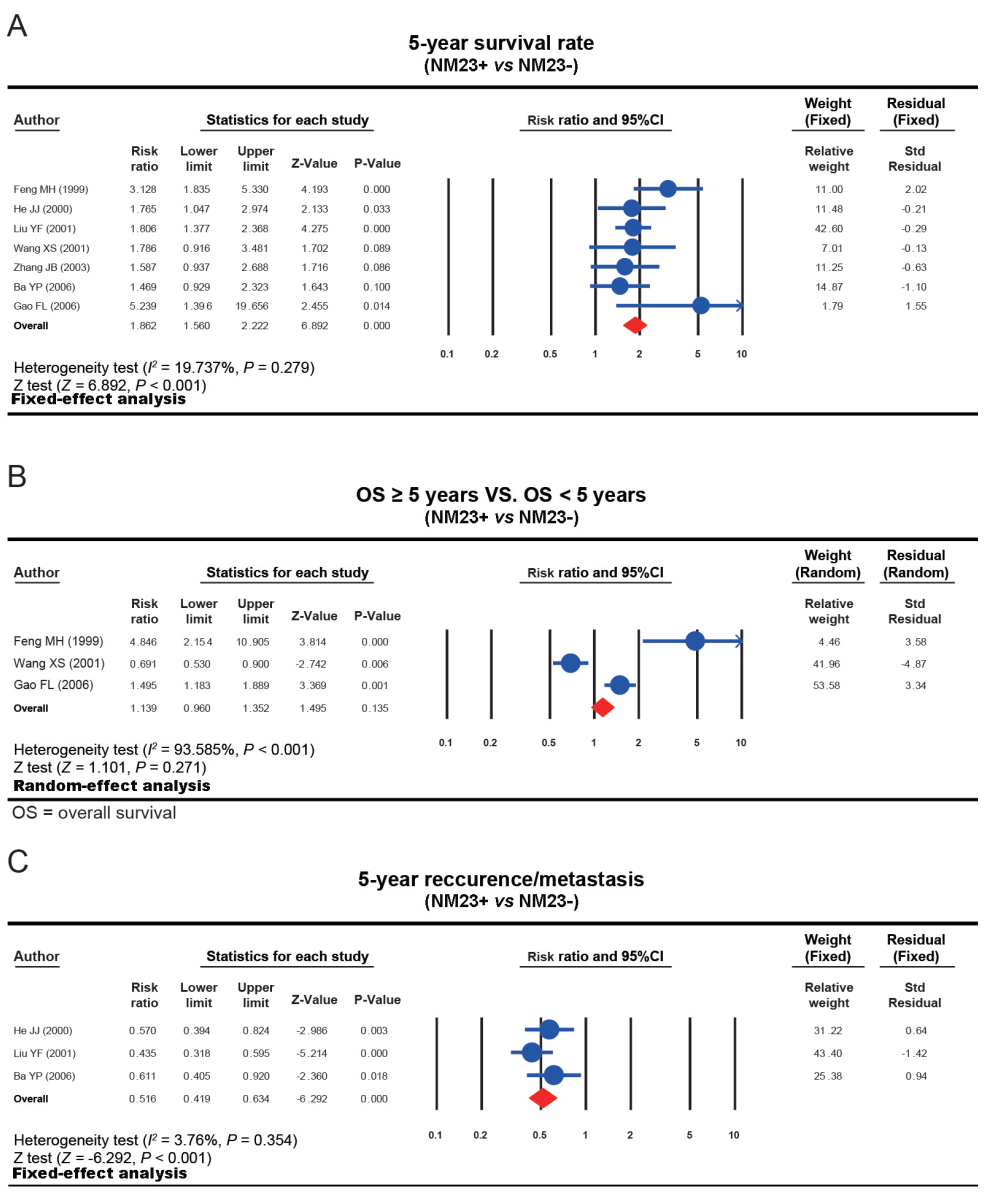

Figure 4. Forest analyses for the correlation between NM23 expression and prognosis of colorectal cancer among Asians. 


\section{Sensitive analysis and publication bias}

Sensitivity analyses showed that no single study significantly affected the overall estimate of the correlation between NM23 expression, tumor pathology, and prognosis of CRC (Figure 5). Moreover, we found no obvious asymmetry in the shape of the funnel plots. The Egger regression test confirmed the absence of publication bias $(P>0.05)$.

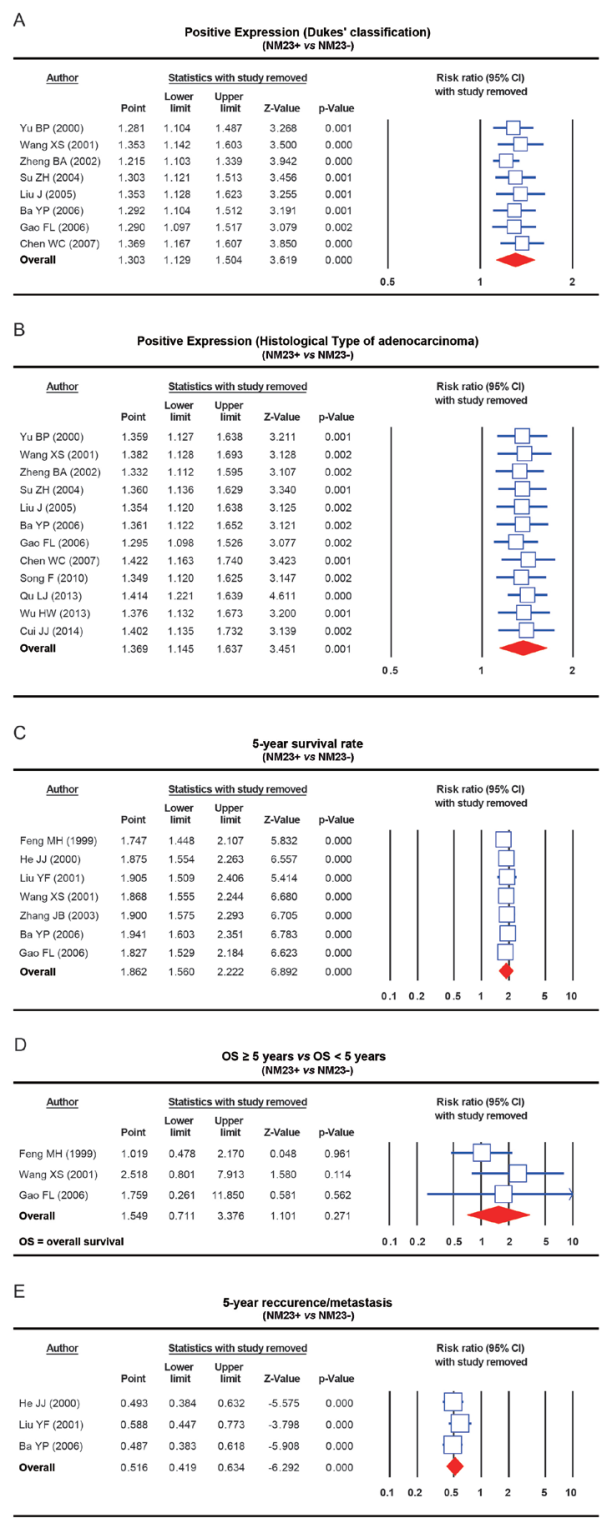

Figure 5. Sensitivity analysis on the correlation between NM23 expression, tumor pathology, and prognosis of colorectal cancer in Asians. 


\section{DISCUSSION}

Meta-analysis of 16 randomized cohort studies revealed that NM23 expression is associated with tumor pathology in CRC in Asians. A higher NM23 protein expression level was observed in patients with Dukes stage A and B than in patients with Dukes stage C and D. The NM23 protein was also expressed at higher levels in moderately differentiated tumors than in poorly differentiated tumors. NM23 is a nucleoside diphosphate kinase and also has other enzymatic activities such as histidine kinase, transcriptional activation, and exonuclease activities (Marino et al., 2012). NM23-H1 is well known for its ability to regulate distinct cellular functions (Lin et al., 2014). NM23 family proteins are involved in multiple-biological processes, such as cell differentiation, adhesion, migration, microtubule polymerization, signal transduction pathway, vascular invasion, and endocytosis, as well as tumor cell shape and apoptosis (Lin et al., 2014). The stage of local confinement of a tumor and the presence or absence of metastases, combined with other specific prognostic and predictive factors, are very important for patient management (Obrocea et al., 2011). Mutations, deletions, and altered expression of NM23 protein family members were found to be closely related to the metastatic capacity of many malignant tumors, including CRC (Wu et al., 2013). There is evidence to support an intracellular role for NM23 in tumor metastasis, such as in CRC (Wu et al., 2013). The NM23 protein is highly conserved from bacteria to humans and contributes substantially to the metastatic process by reducing NM23 protein expression in metastatic lymph nodes, indicating that metastatic tumor cells originate from and are mainly composed of cells with low NM23 expression. Data from several studies included in our meta-analysis showed that NM23 expression levels were correlated with the pathological characteristics of CRC. Our results strongly support that NM23 expression status is associated with the origin and development of CRC in Asians.

Another crucial result in our study is that NM23 expression may be used as a prognostic indicator of CRC among Asians. NM23 was the first identified metastasis suppressor gene and its expression was found to be correlated with decreased metastatic capacity of tumor cells (Marino et al., 2012). The findings of this study also revealed a higher 5-year survival rate in NM23-positive CRC patients than in NM23-negative CRC patients. Five-year tumor relapse and metastasis were observed at a lower rate in NM23-positive CRC patients than in NM23-negative CRC patients, suggesting that NM23 is positive indicator of a favorable CRC prognosis. Previous studies have shown that NM23 protein levels were correlated with the progression of CRC and could be used as an index for predicting invasion and metastasis potential (Liu et al., 2011; Wu et al., 2013). Our results support those of previous studies and further suggest that high NM23 levels are correlated with a better prognosis, and thus NM23 expression can be used as a reliable and independent prognostic indicator in CRC.

There were several limitations in our meta-analysis. Briefly, some of the studies included had relatively small sample sizes. Moreover, selection and language bias may have existed in this metaanalysis because only studies published in English and Chinese languages were used. Furthermore, all 16 studies selected were conducted in China, and thus geographical coverage was limited in identifying ethnic differences among Asian populations when determining the correlation between NM23 expression, tumor pathology, and prognosis of CRC. Further studies should be conducted in other populations to represent a more heterogeneous Asian population to confirm our findings.

In conclusion, we found strong correlations between NM23 expression, tumor pathology, and prognosis of CRC in Asians. Further studies including larger sample sizes focused on Asian populations will contribute to the reliability of our results and validate NM23 as a reliable biomarker for assessing CRC behavior. 


\section{Conflicts of interest}

The authors declare no conflict of interest.

\section{ACKNOWLEDGMENTS}

We acknowledge the reviewers for their helpful comments on this paper.

\section{REFERENCES}

Astin M, Griffin T, Neal RD, Rose P, et al. (2011). The diagnostic value of symptoms for colorectal cancer in primary care: a systematic review. Br. J. Gen. Pract. 61: e231-e243.

Ba YP (2006). Clinical significance of nm23-H1 gene expression in colorectal carcinoma. China Med. Herald 3: 46-47.

Chen WC, Lin MS, Zhang BF, Fang J, et al. (2007). Survey of molecular profiling during human colon cancer development and progression by immunohistochemical staining on tissue microarray. World J. Gastroenterol. 13: 699-708.

Cui JJ, Lu QG and Wang AL (2014). Expression and clinical significances of p53 and nm23 in colorectal carcinoma. J. Colorectal Anal. Surg. 20: 45-47.

Cunningham D, Atkin W, Lenz HJ, Lynch HT, et al. (2010). Colorectal cancer. Lancet 375: 1030-1047.

Feng MH, Wang MN and Wang SF (1999). Association of p53 and nm23-H1 expression and prognosis in colorectal carcinoma without lymphatic metastasis. Chin. J. Exp. Surg. 16: 574.

Gao FL (2006). Correlations of C-erbB-2 and nm23 protein expression with prognosis of rectal cancer. World Chin. J. Digestol. 14: 1015-1018.

He JJ, Chen WK, Yang YJ and Shi JS (2000). The expression and its significance of NM23/NDPK for the patients of large intestinal carcinoma. China J. Modern Med. 10: 35-36.

Higgins JP and Thompson SG (2002). Quantifying heterogeneity in a meta-analysis. Stat. Med. 21: 1539-1558.

linuma H, Watanabe T, Mimori K, Adachi M, et al. (2011). Clinical significance of circulating tumor cells, including cancer stemlike cells, in peripheral blood for recurrence and prognosis in patients with Dukes' stage B and C colorectal cancer. J. Clin. Oncol. 29: 1547-1555.

Jimenez CR, Knol JC, Meijer GA and Fijneman RJ (2010). Proteomics of colorectal cancer: overview of discovery studies and identification of commonly identified cancer-associated proteins and candidate CRC serum markers. J. Proteomics 73: 1873-1895.

John SK, George S, Primrose JN and Fozard JB (2011). Symptoms and signs in patients with colorectal cancer. Colorectal Dis. 13: 17-25.

Lin CH, Dammai V, Adryan B and Hsu T (2014). Interaction between Nm23 and the tumor suppressor VHL. Naunyn Schmiedebergs Arch. Pharmacol. 388: 143-152.

Liu C, Liu J, Wang X, Mao W, et al. (2011). Prognostic impact of nm23-H1 and PCNA expression in pathologic stage I non-small cell lung cancer. J. Surg. Oncol. 104: 181-186.

Liu J, Si C and Li Q (2005). A study on the expression of pKi-67 and nm23 in rectal carcinoma and relationship with the prognosis. Zhejiang J. Prev. Med. 17: 6-10.

Liu YF and Wang Y (2001). Association between $\mathrm{nm} 23-\mathrm{H} 1$ and lymphatic metastasis and prognosis of colorectal carcinoma. Practical Oncol. J. 15: 227-228.

Lu Y, Zhao X, Li K, Luo G, et al. (2013). Thioredoxin-like protein 2 is overexpressed in colon cancer and promotes cancer cell metastasis by interaction with ran. Antioxid. Redox. Signal. 19: 899-911.

Marino N, Nakayama J, Collins JW and Steeg PS (2012). Insights into the biology and prevention of tumor metastasis provided by the Nm23 metastasis suppressor gene. Cancer Metastasis Rev. 31: 593-603.

Marshall JC, Collins J, Marino N and Steeg P (2010). The Nm23-H1 metastasis suppressor as a translational target. Eur. J. Cancer 46: 1278-1282.

Merika E, Saif MW, Katz A, Syrigos K, et al. (2010). Review. Colon cancer vaccines: an update. In Vivo 24: 607-628.

Obrocea FL, Sajin M, Marinescu EC and Stoica D (2011). Colorectal cancer and the 7th revision of the TNM staging system: review of changes and suggestions for uniform pathologic reporting. Rom. J. Morphol. Embryol. 52: 537-544.

Peters JL, Sutton AJ, Jones DR, Abrams KR, et al. (2006). Comparison of two methods to detect publication bias in metaanalysis. JAMA 295: 676-680.

Qu L, Liang L, Su J and Yang Z (2013). Inhibitory effect of upregulated DR-nm23 expression on invasion and metastasis in colorectal cancer. Eur. J. Cancer Prev. 22: 512-522. 
Saha A and Robertson ES (2011). Functional modulation of the metastatic suppressor Nm23-H1 by oncogenic viruses. FEBS Lett. 585: 3174-3184.

Siegel R, Desantis C and Jemal A (2014). Colorectal cancer statistics, 2014. CA Cancer J. Clin. 64: 104-117.

Song F and Gilbody S (1998). Bias in meta-analysis detected by a simple, graphical test. Increase in studies of publication bias coincided with increasing use of meta-analysis. BMJ 316: 471.

Song F, Gao F, Dong L, Zhang X, et al. (2010). Study of the expression and significance of P53 and nm23 in colorectal adenocarcinom. J. Med. Res. 39: 70-72.

Stang A (2010). Critical evaluation of the Newcastle-Ottawa scale for the assessment of the quality of nonrandomized studies in meta-analyses. Eur. J. Epidemiol. 25: 603-605.

Steeg PS, Zollo M and Wieland T (2011). A critical evaluation of biochemical activities reported for the nucleoside diphosphate kinase/Nm23/Awd family proteins: opportunities and missteps in understanding their biological functions. Naunyn Schmiedebergs Arch. Pharmacol. 384: 331-339.

Su ZH and Li JC (2004). Lymphatic metastasis and nm23H1 genetic instability in Chinese colon cancer patients. World J. Gastroenterol. 10: 2800-2804.

Tan HT, Wu W, Ng YZ, Zhang X, et al. (2012). Proteomic analysis of colorectal cancer metastasis: stathmin-1 revealed as a player in cancer cell migration and prognostic marker. J. Proteome Res. 11: 1433-1445.

Wang XS, Xue YW, Dong XS, Liu M, et al. (2001). Clinical significance of p21 and nm23 genes expression and correlated measurement in colerectal carcinoma. Practical Oncol. J. 15: 264-265.

Watson AJ and Collins PD (2011). Colon cancer: a civilization disorder. Dig. Dis. 29: 222-228.

Wu HW, Gao LD and Wei GH (2013). hMSH2 and nm23 expression in sporadic colorectal cancer and its clinical significance. Asian Pac. J. Cancer Prev. 14: 1995-1998.

Yu BP, Wang WA, Yu JP, Shen ZX, et al. (2000). The value of NM23-H1 predication of metastatic potential in colorectal carcinomas. Cancer Res. Prev. Treat. 27: 103-104.

Zhang JB, Chen L, Han F, Yu L, et al. (2003). Overexpression of c-erbB-2 and hypoexpression of nm23 relating to poor prognosis of colorectal carcinoma. Zhonghua Bing Li Xue Za Zhi 32: 115-119.

Zhang JH, Wang LH, Li XJ, Wang AP, et al. (2014). Expression of Ang-2/Tie-2 and PI3K/AKT in colorectal cancer. Asian Pac. J. Cancer Prev. 15: 8651-8656.

Zhen BA, Dong QJ and Deng GL (2002). Clinical significance of p16 and nm23-H1 protein expression in colorectal cancer. Zhejiang Med. J. 24: 462-464.

Zintzaras E and loannidis JP (2005a). Heterogeneity testing in meta-analysis of genome searches. Genet. Epidemiol. 28: 123-137.

Zintzaras E and loannidis JP (2005b). HEGESMA: genome search meta-analysis and heterogeneity testing. Bioinformatics 21: 3672-3673. 